\title{
High-temperature behaviour of average structure and vibrational density of states in the ternary superionic compound AgCuSe
}

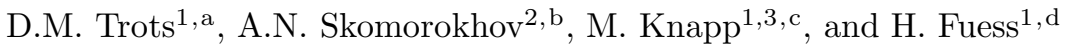 \\ 1 Institute for Materials Science, Darmstadt University of Technology, Petersenstr. 23, 64287 Darmstadt, Germany \\ 2 Institute for Physics and Power Engineering, Bondarenko Sq.1, 249033 Obninsk, Kaluga reg., Russia \\ 3 CELLS, P.O.B 68, 08193 Barcelona, Spain
}

Received 11 February 2006 / Received in final form 27 April 2006

Published online 22 June 2006 - (C) EDP Sciences, Società Italiana di Fisica, Springer-Verlag 2006

\begin{abstract}
Results of simultaneous thermal analysis (STA), synchrotron powder diffraction (in the range $300-973 \mathrm{~K}$ ) and inelastic neutron scattering (at 285 and $505 \mathrm{~K}$ ) on non-superionic $\beta$ - and superionic $\alpha$-AgCuSe are reported. The sample is stable in argon on heating. The volume change at the superionic phase transition is about 5\%. A model for the average structure of $\alpha$-AgCuSe is proposed. No anomalies in the temperature dependence of the parameters of the average structure were revealed. Ionic conductivity in $\alpha$-AgCuSe can originate from cation jumps in "skewed" $\langle 100\rangle$ directions between nearest-neighbour tetrahedral sites via the peripheries of the octahedral cavities. A correlation between the temperature dependence of the cation redistribution in $\alpha$-AgCuSe and the temperature dependence of the ionic conductivity is supposed. Various contributions (anharmonic effects, time-average static disorder and phononphonon scattering) to the widths of individual phonons upon temperature increase lead to pronounced changes in the neutron-weighted densities of states of $\beta$ - and $\alpha$-AgCuSe and accompany the superionic phase transition as well.
\end{abstract}

PACS. 61.43.-j Disordered solids - 61.10.-i X-ray diffraction and scattering - 78.70.Nx Neutron inelastic scattering

\section{Introduction}

For a long time, copper and silver based selenides have attracted the attention of scientists due to their exceptional transport properties. Recently, the high ionic conductivity renewed the practical interest in silver copper selenide, $\mathrm{AgCuSe}$. In the superionic $\alpha$-phase of $\mathrm{AgCuSe}$ the ionic conductivity at temperatures above $473 \mathrm{~K}$ is as high as $1.5 \Omega \mathrm{cm}^{-1}[1,2]$. Superionic conductors find technological applications in energy storage devices and selective gas pressure monitors. Moreover, Balapanov et al. reported very large ionic thermoelectromotive force coefficients in copper- and silver based selenides [3]; therefore an implementation of these materials in thermoelectric devices is of considerable interest [3].

The earliest structural investigation reported a tetragonal structure for $\beta$-AgCuSe at room temperature (RT), space group $P 4 / n m m$, with lattice parameters $a=4.075 \AA$ and $c=6.29 \AA, Z=2$ [4]. Shortly thereafter

\footnotetext{
a e-mail: d_trots@yahoo.com

b e-mail: ans@ippe.ru

c e-mail: mknapp@cells.es

d e-mail: hfuess@tu-darmstadt.de
}

Frueh et al. [5] found that $\beta$-AgCuSe at RT crystallizes in pseudo-tetragonal orthorhombic symmetry and has a large supercell $a \times 5 a \times c$ with $Z=10$ (space group Pmmn) with respect to a tetragonal subcell with lattice parameters $a=4.07-4.105 \AA$ and $c=6.31 \AA$. Moreover, these authors found a reversible phase transition in the hightemperature phase at $463-468 \mathrm{~K}$. Kiazimov et al. determined the equilibrium temperature between the low$(\beta)$ and high-temperature $(\alpha)$ modifications of $\mathrm{AgCuSe}$ at $504 \mathrm{~K}$, i.e. only the cubic phase exists above this temperature $[6,7]$. In more recent reports $\alpha$-AgCuSe was indexed based on a cubic face-centered cell (fcc) with $a=6.080 \AA$ at $523 \mathrm{~K}$. Recently, several additional reflections in the diffraction pattern of $\beta$-AgCuSe at RT were reported [8] which were not listed in the previous literature. Furthermore a large superstructural orthorhombic cell was reported in [9] on the basis of electron diffraction. In the latter work diffraction patterns of $\beta$ - $\mathrm{AgCuSe}$ at RT were indexed based on an orthorhombic cell $a \times 10 a^{\prime} \times 2 c$ with $Z=40$, where $a=4.105 \AA, a^{\prime}=4.070 \AA, c=6.310 \AA$ are the lattice parameters of the basic primitive subcell proposed by Frueh et al. [5]. A pronounced structural disorder in superionic $\alpha$-AgCuSe was reported in [10]. In this 
report the structure at $523 \mathrm{~K}$ was derived from conventional X-ray powder diffraction only. Accordingly, anions are situated on the $4(a)$ site at $(0,0,0)$, whereas cations are statistically distributed between $8(c)$ at $(1 / 4,1 / 4,1 / 4)$ and $32(f)$ sites at $(x, x, x)$ with $1 / 3<x<1 / 2(Z=4$, space group $F m \overline{3} m$ ).

To our knowledge, there are no data on the hightemperature structural evolution of the average structure and the vibrational density of states in $\mathrm{AgCuSe}$ in the literature. In the present work we report the results of diffraction studies on $\mathrm{AgCuSe}$ in the temperature range between 300 and $973 \mathrm{~K}$ and inelastic neutron scattering results at 285 and $505 \mathrm{~K}$.

\section{Experimental details and data reduction}

The AgCuSe sample was synthesized via a solid-state route at $1173 \mathrm{~K}$ in silica tubes, evacuated down to $10^{-3}$ mbar and sealed. Preliminary X-ray diffraction (XRD) measurements were performed using a STOE STADI $\mathrm{P}$ diffractometer $\left(\mathrm{Cu} \quad \mathrm{K} \alpha_{1}\right.$ radiation, curved Ge (111) monochromator, transmission mode, step $0.03^{\circ}$ $(2 \theta)$, curved phase-sensitive detector). In order to characterize the stability of AgCuSe, STA measurements, which combined thermogravimetry (TG) and differential scanning calorimetry (DSC), were carried out on a Netzsch STA 429 device at a heating-cooling rate of $10 \mathrm{~K} / \mathrm{min}$, either in air or in argon.

High-temperature structure investigations in the temperature range RT-973 K were performed at the synchrotron facility Hasylab/DESY (Hamburg, Germany) with powder diffractometer at beam-line B2 [11,12]. A $0.3 \mathrm{~mm}$ quartz capillary was filled with powdered AgCuSe under argon atmosphere and sealed. Subsequently the capillary was mounted inside a STOE furnace in Debye-Scherrer geometry, equipped with a EUROTHERM temperature controller and a capillary spinner. Full patterns were collected over a $2 \theta$ range of $4-40^{\circ}$ with a step size of $0.004^{\circ}$ in temperature steps of $50 \mathrm{~K}$. The final product was analysed at RT after heating to $973 \mathrm{~K}$ and cooling. The wavelength $\lambda=0.470015 \AA$ was determined from 6 reflection positions of the Si SRM640b standard sample. Data evaluation was performed using the program package "FULLPROF" [13]: lattice parameter, zero shift, background and profile shape parameters were refined by a whole-pattern decomposition procedure; the time-averaged structure was analysed by the full-profile Rietveld method with fixed values of already refined parameters.

The inelastic neutron scattering spectra of $\beta$ - and $\alpha$-AgCuSe were collected at 285 and $505 \mathrm{~K}$ with the DIN-2PI direct geometry spectrometer installed at the IBR-II pulsed neutron reactor in Dubna, Russia [14]. In order to reduce sample absorption and to extend the kinematical range, an incident neutron energy of $18.496 \mathrm{meV}$ was selected. A polycrystalline sample (about $30 \mathrm{~g}$ ) was placed in a slab holder $\left(1 \times 5 \times 110 \mathrm{~mm}^{3}\right)$ made from thin aluminium foil and mounted in a sample chamber at an angle of $60^{\circ}$ between the incident beam and the normal of the slab (reflection geometry). The sample chamber was evacuated down to $10^{-2}$ mbar. Neutron scattering data were collected simultaneously at 12 scattering angles in the kinematical range from $1.94 \AA^{-1}$ to $5.50 \AA^{-1}$. The energy resolution of the spectrometer was about $5-8 \%$ for energy transfers between $-13-160 \mathrm{meV}$ (negative values correspond to neutron energy loss). Empty can and vanadium normalization runs were also performed. The standard corrections for the background due to the empty container (taking into account self-shielding of the sample geometry), normalization and detector efficiency corrections were applied to the measured differential cross section data by the standard data reduction procedure at DIN-2PI. Since the absorption cross section of the sample at the selected incident energy was sufficiently large and a thin slab sample holder was used, multiple scattering effects could be neglected [15]. In spite of the mainly coherent scattering from AgCuSe, an estimation of the generalized frequency spectrum is possible even for such a sample. In this case, according to the incoherent approximation [16], coherence effects should be of minor importance if a time-of-flight experiment is performed on a powder sample (directional averaging) with a sufficiently large incident energy (some meV) and in such a large kinematical range. Thus, we employed the incoherent approximation and used as starting point for the derivation of the frequency spectrum the standard phonon expansion (see for example, [17]). The multiphonon correction was performed according to the self-consistent iterative procedure well described in $[17,18]$. Frequency spectra below $2.5 \mathrm{meV}$ were dominated by a large elastic peak due to resolution effects, nevertheless, this peak was easily removed and, finally, data below $2.5 \mathrm{meV}$ were extrapolated to zero transferred energy in Debye-like form.

\section{Results and discussion}

\subsection{Stability of the sample}

After preliminary XRD measurements, which revealed no impurities in the sample, $\mathrm{AgCuSe}$ was characterized by STA (Fig. 1). The DSC curve of AgCuSe measured in air displays a strong endothermic signal at $480 \mathrm{~K}$, which corresponds to the superionic phase transition (Fig. 1a). This is in agreement with calorimetric, XRD and ionic conductivity data from the literature $[1,2,5,7,9,10]$. The TG curve clearly indicates an increase of the sample weight during heating in air. This corresponds to an oxidation of the sample. XRD phase analysis showed decomposition of the initial sample into copper oxide (PDF-2 card [45-937]), silver selenite (PDF-2 card 34-382) and silver (PDF-2 card [89-3722]). A possible oxidation mechanism of $\mathrm{AgCuSe}$ is suggested in [19]. On the other hand, no obvious changes of the sample weight were observed in the TG curve measured in argon (Fig. 1b). XRD analysis of the sample, which was heated in argon during STA, showed no significant changes with respect to the initial state. The DSC heating curve of AgCuSe in argon exhibits two pronounced endothermic peaks with maxima at 480 


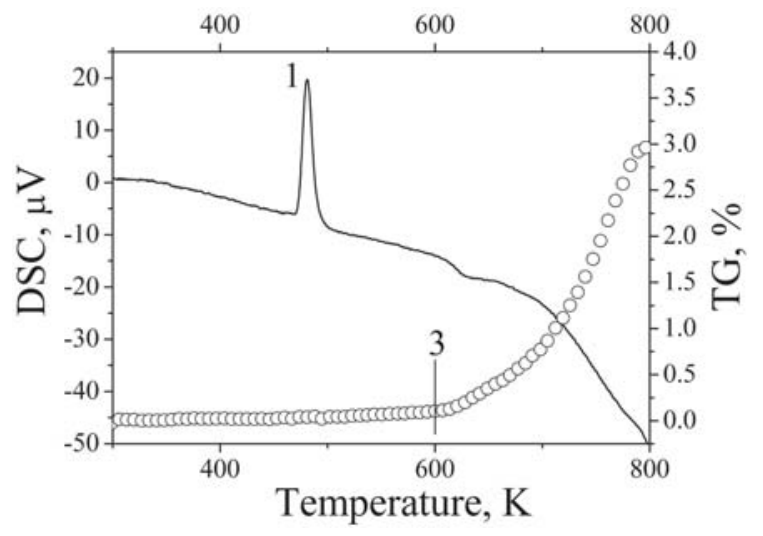

(a)

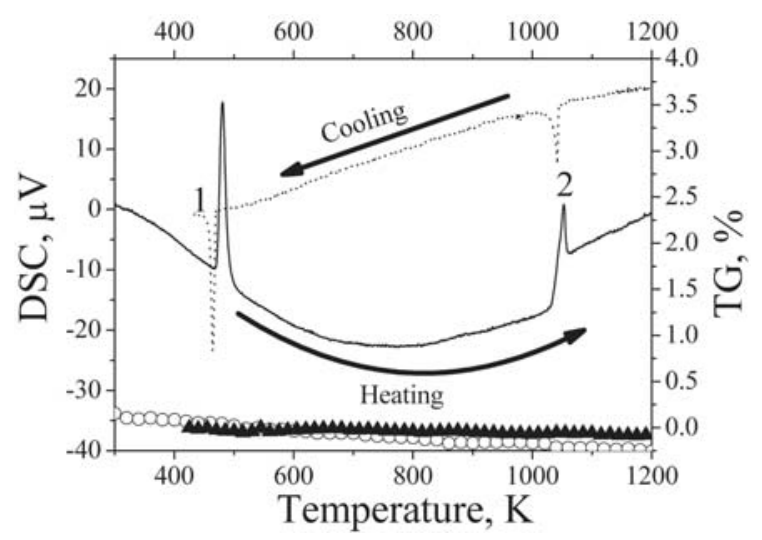

(b)

Fig. 1. STA traces for AgCuSe at a heating-cooling rate of $10 \mathrm{~K} / \mathrm{min}$; (a) in air, (b) in argon. Solid and dotted lines - DSC curves recorded during heating and cooling, open and closed symbols - TG curves at heating and cooling. 1 - peak which corresponds to the superionic phase transition; 2 - peak which corresponds to the melting point; 3 - temperature threshold for oxidation.

and $1054 \mathrm{~K}$, corresponding to the superionic phase transition and melting of the sample, respectively. The DSC data during successive cooling show thermal events at 464 and $1042 \mathrm{~K}$, i.e. some hysteresis in comparison with heating.

\subsection{Structural behaviour of AgCuSe at high temperatures}

In agreement with calorimetric studies, high-temperature synchrotron powder diffraction measurements revealed two phases in the temperature range studied. Attempts to refine the structure of non-superionic $\beta$ - $\mathrm{AgCuSe}$ based on the model from Frueh et al. (ICSD \#44608, [5]) were unsuccessful. Diffraction patterns of $\beta$-AgCuSe were indexed, according to the results from electron diffraction [9], based on a large orthorhombic supercell $a \times 10 a^{\prime} \times 2 c$ with $Z=40$ (space group Pmmn), where $a=4.105 \AA, a^{\prime}=4.070 \AA$, $c=6.310 \AA$ are lattice parameters at RT of the basic orthorhombic primitive subcell proposed by Frueh et al. [5].

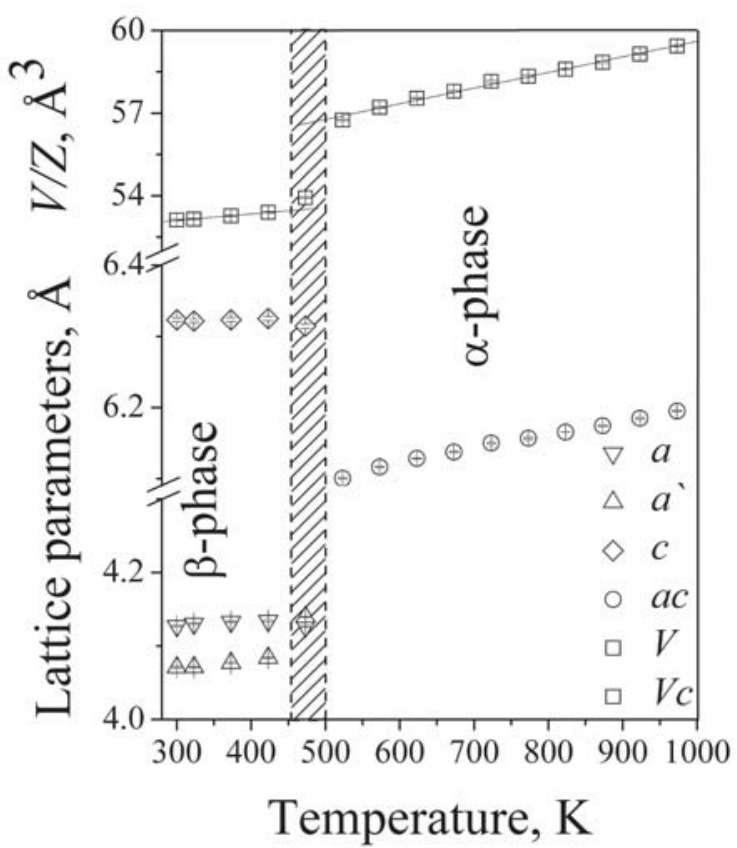

Fig. 2. Temperature dependence of the lattice parameters of the basic orthorhombic subcell of $\beta$-AgCuSe $(a-$ upside-down triangles, $a^{\prime}$-triangles, $c$-diamonds), cubic lattice parameters $a_{c}$ of $\alpha$-AgCuSe (circles) and the unit cell volume per formula unit $(V / Z)$ of $\beta$ - and $\alpha$-AgCuSe (squares). The hatched region indicates the region in which the superionic phase transition takes place (as defined from the DCS curves). The estimated standard deviations are similar as the size of the symbols.

In accordance with other authors $[6,7,9,10]$, the hightemperature phase of silver copper selenide was indexed based on a cubic cell (space group $F m \overline{3} m, a=6.1002(8) \AA$ at $523 \mathrm{~K}$ ). The temperature dependences of lattice parameters and volume per formula unit of $\beta$ - and $\alpha$-AgCuSe are presented in Figure 2. The discontinuous volume change of about $5 \%$ at the superionic $\beta \rightarrow \alpha$ transition is a very strong indication for a 1st order phase transition. The volume thermal expansion coefficients are $44 \times 10^{-6} \mathrm{~K}^{-1}$ and $95 \times 10^{-6} \mathrm{~K}^{-1}$ for the $\beta$ - and $\alpha$-phases, respectively.

We used the so called "split-site" analysis (see for example, [20]) to display disorder in $\alpha$-AgCuSe. During refinement great care was taken to ensure that data were not overinterpreted by models with an inappropriate large number of structural parameters. The cation distribution was parameterised using different structural models with silver and copper cations randomly distributed over tetrahedral and octahedral interstitial sites within a rigid selenium fcc sublattice. Refinements were based on a $F m \overline{3} m$ symmetry and are summarized in Table 1 . The results demonstrate a considerable degree of cation disorder which can be modelled by a random occupation of the tetrahedral $8(c)$ site at $(1 / 4,1 / 4,1 / 4)$ and the octahedral $32(f)$ site at $(x, x, x)$ with $1 / 3<x<1 / 2$ (Figs. 3 and 4$)$. This indicates cation disorder in $\langle 111\rangle$ directions. Attempts were also made to refine the data in the ideal antifluorite structure and in a 'one-site' model with cations 
Table 1. Temperature behaviour of structural parameters of $\alpha$-AgCuSe in the range $523-973 \mathrm{~K}$. Space group $F m \overline{3} m ; Z=4$; atom positions: anions on $4(a)$ at $(0,0,0)$; cations on $8(c)$ at $(1 / 4,1 / 4,1 / 4)$ and $32(f)$ at $(x, x, x)$ with $1 / 3<x<1 / 2$. Refined parameters: isotropic thermal displacement parameters of anions $B$ and cations $B_{1}, B_{2}$; cation position parameter $x$; cation site occupancies $S I T E_{1}$ and $S I T E_{2}$. Constrained thermal displacement parameters are used for $\mathrm{Ag}^{+}$and $\mathrm{Cu}^{+}$; subscripts "1" and "2" denote cations in different "split" positions. Parameter constraints: $B_{1}=B_{2}, S I T E_{1}+S_{T T}=100 \%$.

\begin{tabular}{ccccccc}
\hline Temperature, $\mathrm{K}$ & $\mathrm{R}_{\text {Bragg }} / \mathrm{R}_{\mathrm{F}} / \chi^{2}, \%$ & $a, \AA$ & $B, \AA^{2}$ & $B_{1}, \AA^{2}$ & $x$ & $S I T E_{1}, \%$ \\
\hline 523 & $3.57 / 5.40 / 7.24$ & $6.1002(8)$ & $6.0(4)$ & $12.5(4)$ & $0.419(2)$ & $53.1 \pm 0.8$ \\
573 & $4.80 / 3.26 / 8.14$ & $6.1165(7)$ & $7.4(5)$ & $15.3(5)$ & $0.417(2)$ & $52.8 \pm 1.0$ \\
623 & $5.05 / 3.18 / 8.71$ & $6.1284(7)$ & $7.2(5)$ & $15.2(5)$ & $0.411(2)$ & $51.7 \pm 1.0$ \\
673 & $6.63 / 4.21 / 7.45$ & $6.1375(9)$ & $7.9(5)$ & $15.0(5)$ & $0.405(2)$ & $51.3 \pm 1.0$ \\
723 & $4.85 / 3.00 / 9.84$ & $6.1500(11)$ & $8.2(5)$ & $16.5(5)$ & $0.406(2)$ & $51.5 \pm 1.0$ \\
773 & $8.47 / 5.14 / 8.09$ & $6.1567(14)$ & $8.7(5)$ & $16.8(4)$ & $0.408(2)$ & $52.3 \pm 0.6$ \\
823 & $5.48 / 3.76 / 8.20$ & $6.1655(1)$ & $7.8(6)$ & $16.4(6)$ & $0.405(2)$ & $50.1 \pm 1.3$ \\
873 & $5.91 / 8.33 / 8.95$ & $6.1742(13)$ & $6.9(7)$ & $17.3(5)$ & $0.410(2)$ & $50.2 \pm 1.3$ \\
923 & $7.31 / 4.31 / 10.3$ & $6.1849(13)$ & $8.9(8)$ & $19.8(7)$ & $0.404(2)$ & $51.5 \pm 1.0$ \\
973 & $6.96 / 3.91 / 7.99$ & $6.1950(11)$ & $8.2(5)$ & $21.2(5)$ & $0.410(2)$ & $52.0 \pm 1.0$ \\
\hline
\end{tabular}

disordered in $\langle 111\rangle$ directions at $32(f)$ at $(x, x, x)$ with $1 / 4<x<1 / 3$ sites. Furthermore, the following models with cations distributed between two sites were tested: $8(c)$ at $(1 / 4,1 / 4,1 / 4)$ and $4(b)$ at $(1 / 2,1 / 2,1 / 2)$ site (illustrates cation disorder in $\langle 111\rangle$ directions $) ; 8(c)$ at $(1 / 4,1 / 4,1 / 4)$ and $48(i)$ at $(x, x, 1 / 2)$ with $1 / 3<x<1 / 2$ sites (cation disorder in $\langle 110\rangle$ directions $) ; 8(c)$ at $(1 / 4,1 / 4,1 / 4)$ and $24(d)$ at $(0,1 / 4$, $1 / 4)$ site as well as between $8(c)$ at $(1 / 4,1 / 4,1 / 4)$ and $48(g)$ at $(x, 1 / 4,1 / 4)$ with $1 / 3<x<1 / 2$ sites (cations are disordered in $\langle 100\rangle$ directions). All these models were either unstable in the refinement due to high correlations between fitted parameters or produced a worse fit to the experimental data.

The model with cations disordered between $8(c)$ at $(1 / 4$, $1 / 4,1 / 4)$ and $32(f)$ at $(x, x, x)$ with $1 / 3<x<1 / 2$ yields a temperature dependence for the parameters of the average structure without any anomalies: isotropic temperature factors increase with increasing temperature (the temperature factors of the cations increase more rapidly); the cation positions of the octahedral site $32(f)$ at $(x, x, x)$ with $1 / 3<x<1 / 2$ are slightly shifting from the centre of the octahedron; there are no pronounced changes in the temperature dependence of the cation occupancies (Tab. 1). Within this model, disorder occurs in $\langle 111\rangle$ directions towards the faces of the octahedron rather than to the octahedron edges; in spite of the significant octahedral occupation there is minimum cation density on the ideal octahedral site $4(b)$ at $(1 / 2,1 / 2,1 / 2)$. In view of these crystal structure features, it is likely that cations jump in skewed $\langle 100\rangle$ directions between nearest-neighbour tetrahedral sites via the peripheries of the octahedral cavities. The same mechanism of ionic conductivity was proposed on the basis of powder diffraction studies for the superionic antifluorite phases of $\mathrm{Ag}_{2} \mathrm{Te}[21], \mathrm{Cu}_{2-\delta} \mathrm{Se}(\delta=0$, $0.15,0.25)[22]$ and is obvious from analysis of diffraction results on $\mathrm{CuI}[23]$ and $\mathrm{Cu}_{1.95} \mathrm{~S}$ [24]. Note that diffraction results on a number of copper- and silver based halides and chalcogenides (for instance, $[22,25,26]$ ) imply that ionic conductivity can be sensitive to details of the cation redistribution between available interstitial sites vs. temperature or the stoichiometry of samples. Taking this

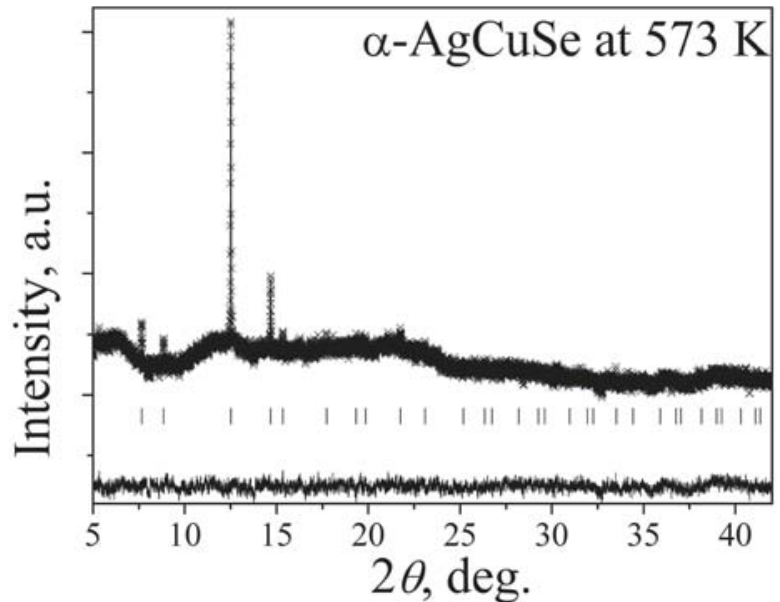

(a)

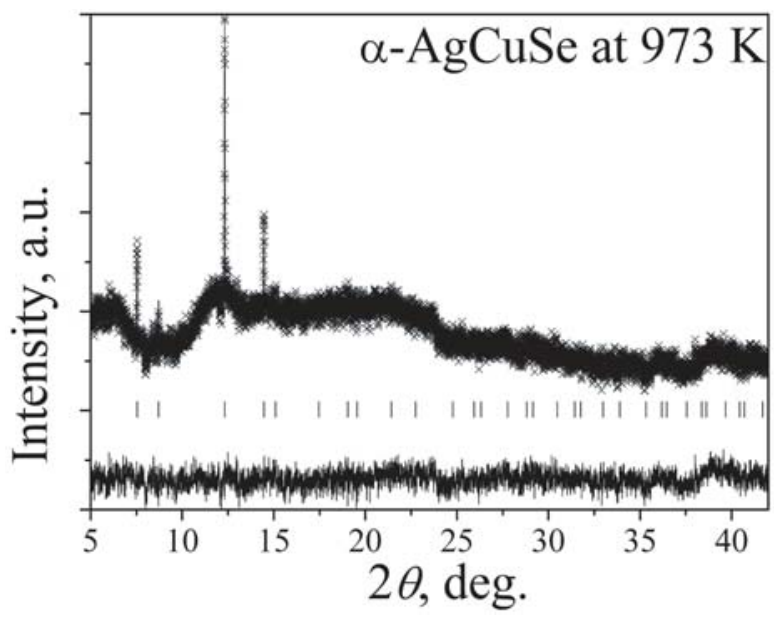

(b)

Fig. 3. Synchrotron powder diffraction patterns of $\alpha$-AgCuSe at 523 and $973 \mathrm{~K}(\lambda=0.470015 \AA)$. The Rietveld fit is based on the model with cations on tetrahedral $8(c)$ and octahedral $32(f)$ sites (Tab. 1). Crosses are experimental data, the line through the crosses is the calculated profile and the lower curve their difference. Tick marks show the calculated positions of $\alpha$-AgCuSe reflections. 


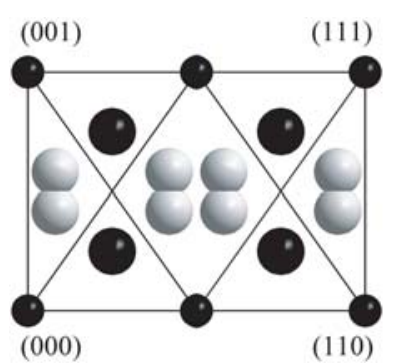

(a)

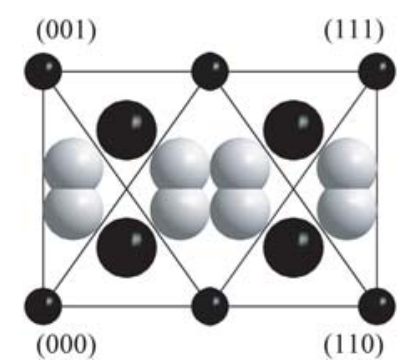

(b)

Fig. 4. Schematic representation of the average structure of $\alpha$-AgCuSe at $523 \mathrm{~K}$ (on the top) and $973 \mathrm{~K}$ (on the bottom). Plots show the thermal ellipsoids at the $50 \%$ probability level for all atoms in a (110) section through the unit cell. Small dark spheres: anions on the $4(a)$ sites at $(0,0,0)$, large dark spheres: cations on the ideal tetrahedral $8(c)$ sites at $(1 / 4,1 / 4$, $1 / 4)$, bright spheres: cations on the octahedral $32(f)$ sites at $(x, x, x)$ with $1 / 3<x<1 / 2$. The plots show $100 \%$ of occupancy for all crystallographically allowed cation positions. The diagonal lines indicate the positions of the boundaries between tetrahedral and octahedral interstices.

into account, we can propose a correlation between the results of the electrochemical measurements from Miyatani et al. [1,2], which revealed only a relatively modest increase in ionic conductivity with temperature within $\alpha$-AgCuSe, and our diffraction results, which show no pronounced cation redistribution. The same correlation was reported for superionic fcc- $\mathrm{Ag}_{x} \mathrm{~Pb}_{1-x} \mathrm{I}_{2-x}$ with $x=1 / 3$ and $2 / 3[25]$.

\subsection{Generalized neutron-weighted densities of states in $\beta$ - and $\alpha$-AgCuSe}

Neutron-weighted densities of states, $Z(\varepsilon)$, for both phases studied are presented in Figure 5 . The spectra of $Z(\varepsilon)$ in $\beta$ and $\alpha$-AgCuSe can be described as a superposition of two well-defined broad peaks at an energy transfer $\varepsilon$ of about 8 and $18 \mathrm{meV}$. The linear (instead of a square) dependence of $Z(\varepsilon)$ in the energy transfer range of $2.5<\varepsilon<5 \mathrm{meV}$ for both phases is particularly noteworthy. Such non-Debye behaviour of $Z(\varepsilon)$ at low energies is an evidence of the high level of anharmonicity in the studied system.

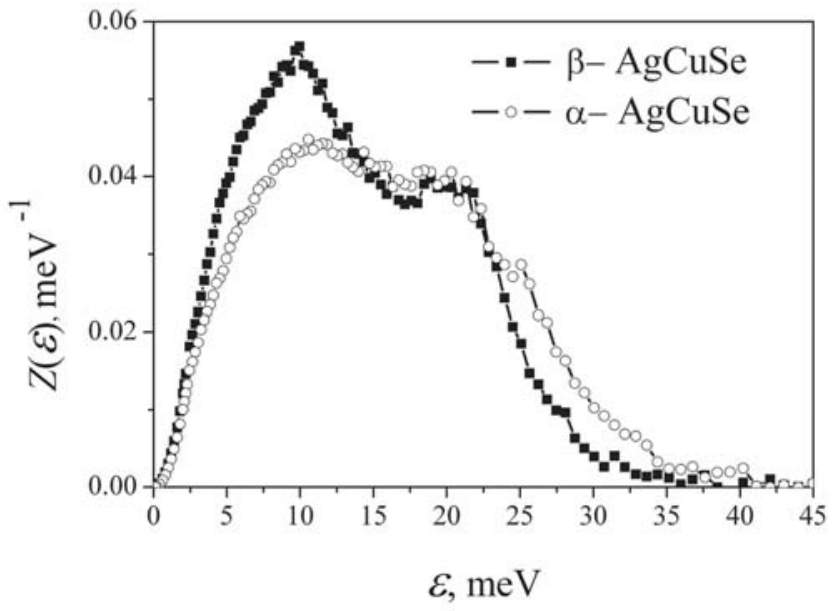

Fig. 5. Neutron-weighted density of states of non-superionic silver copper selenide at $285 \mathrm{~K}$ (filled squares) and superionic at $505 \mathrm{~K}$ (empty circles).

The temperature effects usually manifest in a phononic broadening [27]. Such a phonon broadening in superionics is commonly attributed to increasing anharmonic effects and time-averaged static disorder (see for example, $[27,28])$. Moreover, increasing phonon-phonon scattering due to increasing phonon occupancies at higher temperatures play a crucial role in phonon broadening (see for example, [29]). It appears that this phonon broadening is reflected in the phonon density of states as a general smearing out of well defined features and a high-energy cut-off. Therefore, apparent changes in the frequency spectrum of superionic $\alpha$-AgCuSe such as a damping of the low-energy part (peak at $\sim 8 \mathrm{meV}$ ) and smearing of the high-energy part (peak at $\sim 18 \mathrm{meV}$ ) of the spectrum are clearly visible compared to the spectrum of non-superionic $\beta$-AgCuSe. Such changes in the frequency spectrum of the $\alpha$-phase cannot only arise because of the temperature effects on phonons mentioned above, in the present case they are accompanied with a 1st order superionic phase transition as well. Moreover, the noticeable excess in the density of states in superionic $\alpha$-AgCuSe above $23-25 \mathrm{meV}$ is clearly visible. Such an effect arouses suspicion, because the corresponding range lies above the thermal energy, $k T$, for the $\beta$-AgCuSe and these phonon states were not thermally populated, while the measurements were performed in upscattering (phonon annihilation) regime, i.e. this effect can stem from the thermal population of these phonon states in $\alpha$-AgCuSe. Nevertheless, it should be noted that the dependence of the inelastic intensity in the upscattering regime is usually influenced by instrumental or thermal effects in those kinds of neutron experiments. In our case, these effects were taken into account during data analysis by implementation of the thermal population factor and a multiphonon correction procedure. Nevertheless, in [18] it is shown that smearing of the high-energy part of the spectra - above $23-25 \mathrm{meV}$ in our case - can probably be nothing else but a mathematical problem of data reduction during subtracting of the multiphonon effects. Hence, in our opinion, the excess of the density of states 
above $23-25 \mathrm{meV}$ in $\alpha$-AgCuSe is rather a mathematical problem of the data reduction during the subtraction of the multiphonon terms than a physical effect. However, the description of the self-consisting iterative procedure as well as the weaknesses of such a correction is not the topic of this paper and can be found elsewhere (see for instance [18]).

\section{Summary}

The large volume change $(5 \%)$ at the superionic phase transition accompanied with the abrupt increase of ionic conductivity show that AgCuSe undergoes a type-I superionic transition according to the classification of Boyce and Huberman [30]. A model for ionic conduction in $\alpha$-AgCuSe was derived by synchrotron powder diffraction data: cations jumps in "skewed" $\langle 100\rangle$ directions between nearest-neighbor tetrahedral sites via the peripheries of the octahedral cavities. No anomalies in the temperature dependence of the average structure were observed. Doubtlessly, single crystal measurements of $\alpha$-AgCuSe would provide more information about the underlying structure. Such measurements are, however, practically impossible (or at least extremely difficult) even if single crystals are available, since the 1st order superionic phase transition will lead to fracture. The temperature dependence of cation distribution in $\alpha$-AgCuSe as described by our structural model correlates well with the temperature behaviour of ionic conductivity and gives strong support for the validity of the model.

The neutron-weighted density of states show a nonDebye-like behaviour for $\mathrm{AgCuSe}$ in the low-energy region accessed in our experiment. Pronounced changes with temperature in the frequency spectra of $\beta$ - and $\alpha$-AgCuSe result from numerous contributions (increasing anharmonic effects, time-averaged static disorder and phononphonon scattering) and accompany the superionic phase transition as well. In principle, the separation of different contributions to the phonon widths is possible by inelastic coherent neutron scattering on single crystals (see for instance, [27]) which, however, is practically impossible in the case of $\mathrm{AgCuSe}$. Moreover, as we can see in [31], molecular dynamics simulations are the most appropriate method for the investigation of highly anharmonic solids. Still, the central problem of those simulations is to derive a realistic potential, in the sense, that the potential reproduces the experimentally determined density of states within acceptable limits [31]. Hence, our experimental results on the density of states in AgCuSe could be an experimental base for further computational modelling on this highly anharmonic system.

Financial support from the Bundesministerium fuer Bildung und Forschung is gratefully acknowledged. We are also indebted to Dr. N.N. Bickulova for helping with the preparation of the samples, C. Fasel for performing the STA measurements, Dr. V.A. Semenov and V.M. Morozov for assistance with the INS measurements. We are also extremely grateful to Dr. A.T.
Senyshyn and Dr. H. Ehrenberg for the useful discussions and P. Erhart for assisting in the preparation of the manuscript.

\section{References}

1. S. Miyatani, J. Phys. Soc. Jpn 34, 423 (1973)

2. S. Miyatani, Y. Miura, H. Ando, J. Phys. Soc. Jpn 46, 1825 (1979)

3. M.H. Balapanov, R.A. Yakshybaev, U.H. Muhamedianov, Fiz. Tverd. Tela 45, 604 (2003)

4. J.W. Earley, Am. Mineral. 35, 337 (1950)

5. A.J. Frueh, G.K. Czamanske, Ch. Knight, Z. Kristallogr. 108, 389 (1957)

6. Sh.K. Kiazimov, G.Sh. Gasanov, Yu.G. Asadov, Doklady Akademii Nauk Azeibatzhanskoi SSR 11, 33 (1986)

7. Sh.K. Kiazimov, K.M. Jafarov, Yu.G. Asadov, Phase Transit. 21, 11 (1990)

8. T. Ohtani, K. Maruyama, K. Ohshima, Mater. Res. Bull. 32, 343 (1997)

9. K. Chrissafis, N. Vouroutzis, K.M. Paraskevopoulos, N. Frangis, C. Manolikas, J. Alloy. Compd. 385, 169 (2004)

10. T. Shimoyama, M. Arai, T. Sakuma, in 1st International Meeting on Superionic Conductor Physics (Kyoto, 2003)

11. M. Knapp, V. Joco, C. Baehtz, H. Brecht, A. Berghaeuser, H. Ehrenberg, H. von Seggern, H. Fuess, Nucl. Instrum. Methods A 521, 565 (2004)

12. M. Knapp, C. Baehtz, H. Ehrenberg, H. Fuess, J. Synchrotron Rad. 11, 328 (2004)

13. http://www-llb.cea.fr/fullweb/powder.htm

14. Yu.V. Taran, User Guide. Neutron Experimental Facilities at JINR (Dubna, JINR Press, 1992)

15. E.J. Lindley, J. Mayers, in Neutron Scattering at a Pulsed Source, edited by R.J. Newport, B.D. Rainford, R. Cywinski (Adam Hilger, Bristol and Philadelphia, 1988)

16. V.S. Oskoskii, Sov. Phys. Solid State 9, 420 (1967)

17. J. Dawidowski, F.J. Bermejo, J.R. Granada, Phys. Rev. B 58, 706 (1998)

18. J. Wuttke, M. Kiebel, E. Bartsch, F. Fujara, W. Petry, H. Sillescu, Z. Phys. B 91, 357 (1993)

19. V.L. Barone, I.L. Botto, I.B. Schalamuk, Latin. Am. Appl. Res. 33, 1 (2003)

20. D.A. Keen, J. Phys.: Cond. Matter 14, R819 (2002)

21. D.A. Keen, S. Hull, J. Phys.: Cond. Matter 10, 8217 (1998)

22. A. Skomorokhov, D. Trots, M. Knapp, N. Bickulova, H. Fuess, J. Alloy. Compd. (in press)

23. D.A. Keen, S. Hull, J. Phys.: Cond. Matter 7, 5793 (1995)

24. M. Oliveria, R.K. McMullan, B.J. Wuensch, Solid State Ionics 28-30, 1332 (1988)

25. S. Hull, D.A. Keen, P. Berastegui, Solid State Ionics 147, $97(2002)$

26. D.A. Keen, S. Hull, A.C. Barnes, P. Berastegui, W.A. Crichton, P.A. Madden, M.G. Tucker, M. Wilson, Phys. Rev. B 68, 014117 (2003)

27. S.M. Shapiro, F. Reidinger, in Physics of Superionic Conductors, edited by M.B. Salamon (Springer-Verlag, Berlin-Heidelberg-New York, 1979)

28. K. Schmalzl, D. Strauch, H. Schober, Phys. Rev. B 68, 144301 (2003)

29. P.D. Bogdanoff, B. Fultz, J.L. Robertson, L. Crow, Phys. Rev. B 65, 014303 (2001)

30. J.B. Boyce, B.A. Huberman, Phys. Rep. 51, 189 (1979)

31. J.X.M. Zheng-Johansson, I. Ebbsjö, R.L. McGreevy, Solid State Ionics 82, 115 (1995) 